\title{
Short term forecasting of national growth
}

\author{
Antonio Frenda \\ Rome (Italy) \\ Received 24 October 2012; Accepted 5 November 2012 \\ Copyright (c) 2012, Journal Afrika Statistika. All rights reserved
}

Italian National Statistical Institute - Division for National Accounts, Via A. Depretis 74/B - 00184

\begin{abstract}
The contribution of this paper is to produce national smoothed growth indicators that describe the behaviour of economic activity at a monthly frequency, while utilizing a wide range of economic time series. New Eurocoin indicator, that is published monthly by the Bank of Italy, provides a summary index of the medium to long-run component (MLRG) of the gross domestic product (GDP) growth rate and only for the entire aggregate Euro area, by using the Generalized Dynamic Factor Model. The innovation of this research are some procedures, based on Eurocoin approach, to estimate MLRG in countries belonging to Euro Area.

Résumé. Dans cet article, on produit des indicateurs nationaux de la croissance lissée, qui décrivent le comportement de l'activité économique avec une fréquence mensuelle, tout en utilisant un grand ensemble de séries chronologiques macroéconomiques. L'indicateur New Eurocoin, qui est publié tous les mois par la Banque d'Italie, fournit un indice synthétique de la composante à moyen-long terme (MLRG) du taux de croissance concernant le produit intérieur brut (PIB), en utilisant le Modèle à Facteurs Dynamiques Généralisé. La contribution de cette recherche consiste en certaines procédures, basées sur l'approche Eurocoin, pour estimer la MLRG dans les pays qui appartiennent à la zone Euro.
\end{abstract}

Key words: Medium to long run component of the growth (MLRG); National and European common factors; Real time performance.

AMS 2010 Mathematics Subject Classification : $91 B 62$.

\section{Introduction}

New Eurocoin (NE) is a statistics measure of the Euro-Area conjuncture. Estimations of $\mathrm{NE}$ are obtained through the generalized dynamic factor model. The specific references are Altissimo et al. (2001, 2010), Forni et al. (2001, 2004, 2005). The main empirical contribution of this research is to propose some procedures to estimate the following disaggregated 
A. Frenda, Journal Afrika Statistika, Vol. 7, 2012, pages 459-473. Short term forecasting of national growth.

components of European GDP: MLRG concerning some countries belonging to the Euro Area.

Our procedures are based on the Eurocoin methodology in order to obtain smoothing of a stationary time series, therefore avoiding the occurrence of end-of-sample deterioration. We build real-time monthly GDP estimates purified from seasonal and short run fluctuations. In fact, it is a well-known result in literature that band-pass filters could deteriorate at the end of the sample. We focus on medium to long-run component of the growth (MLRG), i.e. the smoothed component of the GDP growth rate obtained by removing the fluctuations of a period shorter than or equal to one year, and it bears no relationship to any definition of trend. A National Eurocoin is built because a national flash estimate of GDP is released by Eurostat about six weeks after the end of the reference quarter. A national indicator can produce real-time monthly estimate of underlying quarterly growth trends. Today, the Eurocoin is only used to outline the whole aggregate Euro Area growth Frenda (2011, 2012). The Eurocoin approach is therefore used in this paper to estimate monthly smoothed GDP components for the following reasons:

1. Euro Area flash estimate is released on a quarterly frequency, with a certain delay and may be subject to significant revisions afterwards.

2. GDP growth in any quarter depends on seasonal effects and measurement errors; therefore GDP can be misleading.

3. GDP can be influenced by factors affecting only a particular sector or country. These factors are not important for outlining the health of the euro area economy as a whole, but they can be used to asses the country's national cycle.

Furthermore, It is a well-known result in the literature isolating the business cycle in integrated series that band-pass filter could deteriorate at the end of the sample, being less reliable for the most recent data. This is very relevant for economic policy. Altissimo et al. (2010) show that the same problem arises with application to stationary time series. And, through New Eurocoin, they develop a method to obtain smoothing of a stationary time series so as to avoid the occurrence of end-of-sample deterioration and short-run fluctuations. In the dynamic factor model approach, a vector of " $n$ " time series is decomposed into two mutually orthogonal components: a common component characterized by few common factors or latent shocks, and a component "idiosyncratic", led by n specific shocks (one for each variable in the panel). These models allow a net reduction of the cross-sectional size of the dataset. We develop monthly smoothed growth indicators for the main Euro Area economies that can utilize a wide range of economic information in real time. We build these national indicators, by the projection of national or Euro Area GDP on European and national factors. Therefore, we build two types of "common factors" (Altissimo et al., 2010):

- "European factors" that are a linear combination of the 157 variables (belonging to different Euro Area countries);

- "National factors" that are a linear combination of the national variables included in our dataset (e.g., for France we have 23 macroeconomic variables that are used to construct the set of regressors useful for the estimation of past and future MLRG values) concerning a specific European country.

Journal home page: www.jafristat.net 
This goal has been achieved for the following countries: Belgium, Italy, France, Germany, Spain. A national Eurocoin (NE) is the projection of bandpassed GDP on a set of regressors, which are linear combination of national variables contained in the Thomson Financial Datastream used by the Bank of Italy. We show that the real time performance is a reasonable approach for the examination of estimate accuracy. The latter sample will be used to build pseudo estimates by a recursive or a rolling window. We test our models using pseudo real time estimates at the end of sample. We compare in this paper real-time indicators with respect to a measure of the "trend-cycle GDP growth" obtained in the middle of the sample by a band pass bilateral filter on GDP growth (i.e. our target).

\section{Medium to Long-Run Growth of Euro Area National GDP: Band-Pass Filter Approach}

Following Altissimo et al. (2010), medium to long-run growth of economic activity can be calculated by removing any fluctuations from real GDP quarterly growth, of a period shorter than or equal to one year. National MLRG is defined considering the spectral decomposition of $y_{t}^{s}$, real GDP quarterly growth for the main economic countries in the aggregate Euro Area. Assuming stationarity $y_{t}^{s}$ can be represented as the sum of sine and cosine waves with different weights, with $\beta(L)$ that is a low-pass filter excluding short waves with a frequency equal to or higher than $\pi / 6$, that corresponds to a period of 1 year. Using the bandpass filters (see e.g. Baxter and King, 1999 and Christiano and Fitzgerald, 2003), our medium to long run components $c_{t}^{s}$, for each sector, is the following symmetric, infinite, two sided linear combination of the national GDP growth series:

$$
c_{t}^{s}=\beta(L) y_{t}^{s}=\sum_{k=-\infty}^{\infty} \beta_{k} y_{t-k}^{s}, \quad \beta_{k}=\left\{\begin{array}{cll}
\frac{k \pi / 6}{k \pi} & \text { for } & k \neq 0 \\
1 / 6 & \text { for } & k=0
\end{array}\right.
$$

Then, $y_{t}^{s}$ has the following decomposition:

$$
y_{t}^{s}=c_{t}^{s}+s_{t}^{s} \beta(L) y_{t}^{s}+[1-\beta(L)] y_{t}^{s}
$$

Since $\beta(1)=1$ the mean $\mu$ of $y_{t}^{s}$ is retained in $c_{t}$ while the mean of the excluded part $s_{t}^{s}$, that is the short-run component of the national GDP growth, is equal to zero. It is necessary to point out that our ideal target, being an infinite moving average, is unobservable. Therefore, since the data on national GDP are finite, equation (1) cannot be applied in practice. So, a finite-sample version of the band-pass filter is developed in section 1.3 (see equation 7 below).

\section{The Generalized Dynamic Factor Model Approach}

In the New Eurocoin (NE) approach, our national indicators smooth stationary series by using only contemporaneous values of a large dataset and they are obtained as a linear combination of the smooth factors: the latent factor are "smooth factors", which are generalized principal components of current values of the variables in the dataset. These factors are designed to remove short-run and variable-specific sources of fluctuation. The Eurocoin construction is based on Generalized Dynamic Factor model, using leading variables in the 
dataset as proxies for missing future values in the variable of interest. The removal of shortterm dynamics of stationary series from the medium to long-run growth can be obtained through band-pass filters that are infinite moving averages and can deteriorate at the end of the sample. NE is obtained as a linear combination of a small number of "smooth factors", which are generalized principal components of current values of the variables in the dataset, and they are designed to remove short-run and variable-specific sources of fluctuation. Since only current values of the variables are used, no end-of-sample deterioration occurs. The generalized dynamic factor model (GDFM), on which Eurocoin indicator is based, must have two characteristics: it must be dynamic, because business cycle questions are typically non-static. Secondly, it must allow for cross-correlation among idiosyncratic components, as orthogonality is an unrealistic assumption for most applications. An important feature of this model is that the common component is allowed to have an infinite moving average (MA) representation, so as to accommodate for both autoregressive (AR) and MA responses to common factors. Dynamic factor model is more general than a static-factor model in which lagged factors are introduced as additional static factors, since AR responses are ruled out in such a model. The main theoretical tool in this context is Brillinger's theory of dynamic principal components (Brillinger, 1981). In a classic factor model, considering the scalar time series variable $Y_{t}$ to forecast and let $X_{t}$ be the N-dimensional time series of candidate predictors, it is assumed that $\left(X_{t}, Y_{t+h}\right)$ admits a factor model with $\mathrm{r}$ common latent factors $F_{t}$ :

$$
\begin{gathered}
X_{t}=\Lambda F_{t}+\varepsilon_{t} \\
Y_{t+h}=\beta_{F}^{\prime} F_{t}+\beta_{\omega}^{\prime} \omega_{t}+\varepsilon_{t+h}
\end{gathered}
$$

where $\varepsilon_{t}$ is an $N \times 1$ vector of idiosyncratic disturbances, $h$ is the forecast horizon, $\omega_{t}$ is an $m \times 1$ vector of observed variables (e.g., lags of $Y_{t}$ ) useful, with $F_{t}$, to forecast $Y_{t+h}$. When the idiosyncratic disturbances $\varepsilon_{t}$ are temporarly iid and cross-sectionally independent, we can consider the equation above as a classic model of factor analysis. Eurocoin is obtained as a projection of the bandpassed GDP (see equation 3) on factors by a generalization of the principal components concept (D'Ambra and Sarnacchiaro, 2010). Each series contained in the dataset for the calculation of Eurocoin is obtained as follow

$$
x_{i t}=\chi_{i t}+\xi_{i t}=b_{i 1}(L) u_{1 t}+b_{i 2}(L) u_{2 t}+\ldots+b_{i q}(L) u_{1 q}+\xi_{i t}
$$

with a common component $\chi$ and an idiosyncratic $\xi$, that are orthogonal at all lead and lags. Eurocoin is an alternative estimate to $c_{t}$, the medium to long run component of GDP.

In this paper, we build a "national Eurocoin". The value of the national $c_{t}^{s}$, with the coefficients $A_{i}$, at the end of the sample, with $t=T$, is so estimated ${ }^{1}$ :

$$
\hat{c}_{T}^{s}=A_{1} F_{1 T}+A_{2} F_{2 T}+\ldots+A_{m} F_{m T} .
$$

So, our indicators are obtained by the projection of $c_{t}^{* s}$ (the national bandpassed GDP) on a set of regressors, which are linear combination of the variables contained in the Thomson Financial Datastream used by the Bank of Italy. Different filtering methods are used in the

\footnotetext{
${ }^{1}$ See New Eurocoin . A tutorial Note; http://eurocoin.bancaditalia.it4
} 
literature both to remove short-term dynamics of stationary series and for analysing the medium to long-run growth (MLRG); the following are compared in this research:

1. Filters based on spectral representation (i.e. bandpass filters);

2. Methods based on generalized dynamic factor models (i.e. Eurocoin Methodology).

In this paper, we are interested to the performance of our disaggregated indicators $\hat{c}_{t}^{s}$ with respect to a measure of the "trend-cycle GDP growth" $c_{t}^{* s}$ obtained in the middle of the sample by a band pass bilateral filter on GDP growth, that is our target.

Our "National Eurocoin" is a real time estimate of the medium to long run component of GDP growth, and the latter is observable, although with a long delay. Therefore, the performance of this indicator and the ones concerning the disaggregated models that we build in this paper (by implementing Eurocoin approach) can be measured. More precisely, the value of the target (the bandpassed data) is available with good accuracy only at time $T+h$, for a suitable $h$. Therefore, our indicators produced at time $T$ can be compared with the target at $T$ produced at time $T+h$. Differently from bandpass filters, that are based on past and future values of GDP and can therefore deteriorate at the end of the sample, when the missing values useful to describe the cycle is replaced by predictions, our disaggregated and national indicators are reliable for most recent data, very relevant for economic policy.

\section{National Indicators: Real Time Experiment}

In this section we outline some national indicators explaining the medium to long run component of GDP by pseudo real time simulations. We dispose of a dataset consisting of 157 monthly macroeconomic variables during the period between January 1987 and March 2011. The main blocks of macroeconomic indicators are as follows (see Table 1):

\begin{tabular}{l|c} 
Data Source & Variables \\
\hline \hline Surveys & 31 \\
Leading Indicators & 6 \\
Demand Indicators & 12 \\
Industrial Production & 32 \\
Wages Indicators & 2 \\
Employment Indicators & 5 \\
Producer Price Index & 26 \\
Exchange rates & 3 \\
Imports-Exports & 8 \\
Money Supply & 8 \\
STANDARD \& POOR'S INDEX & 7 \\
(Italy, Germany, USA, UK) SPREAD & 10 \\
Benchmark Bond & 7 \\
\hline \hline TOTAL & 157 \\
\hline
\end{tabular}

Table 1. Variables used in Estimation by Data Source. 
We focus on medium to long-run components of the growth (MLRG), i.e. the smoothed components of GDP growth rate obtained by removing the fluctuations of period shorter than or equal to one year, and it bears no relationship to any definition of trend. We observe, in the Table 2 below, that Germany is the most represented country in our Dataset, while Netherlands is the least.

\begin{tabular}{l|c} 
Geographic Area & Variables \\
\hline \hline Belgium & 14 \\
Finland & 2 \\
France & 23 \\
Germany & 39 \\
Greece & 1 \\
Italy & 22 \\
Netherlands & 5 \\
Spain & 25 \\
UK & 4 \\
USA & 7 \\
Euro Area & 15 \\
\hline \hline TOTAL & 157 \\
\hline
\end{tabular}

Table 2. National variables contained in Thomson Datastream.

Our elaborations are based, in this research, on the GDP expressed in constant prices. The national models are tested by pseudo real time simulations. Our goal is achieved by the generalized dynamic factor models for the following countries: Belgium, Italy, France, Germany, Spain, Netherlands. Three different types of national indicators can be obtained by the strategies (methods) below indicated:

1. Projection of national GDP on European factors (so that we can use all the 157 national and European variables used by the Bank of Italy to build Eurocoin indicator);

2. Projection of Euro Area GDP on factors built by national variables (e.g. projection of European GDP on the common factors that are linear combination of the 23 French variables included in our Dataset);

3. Projection of national GDP on common national factors (e.g. projection of French GDP on French common factors)".

Our elaborations are based in this research on the GDP by country expressed in constant prices. Data concerning national quarterly gross value added are available from Eurostat Statistics Database by themes. According to Altissimo et al. (2010), within the 2002-2010 finite sample we consider the following approximation of the target (see equation 1) that can be obtained by augmenting the national growth rate $y_{t}^{* i}$ for each country $i$, with its sample mean $\hat{\mu}$ in both infinite directions:

$$
c_{t}^{* i}=\beta(L) y_{t}^{* i}, \text { where } y_{t}^{* i}= \begin{cases}y_{t} & \text { if } \quad 1 \leq t \leq T \\ \hat{\mu} & \text { if } t<1 \text { or } t>T\end{cases}
$$


Since $y_{t}^{s}$, the national growth rate, is observed only quarterly, while we are interested in a monthly indicator of economic activity, we chose a simple interpolation to calculate the two missing points for each quarter, assuming that $y_{t}$ is unchanging within a quarter. Equation (7) provides a good approximation to the ideal target at time $t$ in the middle of the sample, and it performs badly at the beginning and end of the sample. Precisely, the performance of the national Eurocoin at time $t$, with $t \leq T-12$, will be measured as the difference between our indicator at time $t$ and the approximate target at $t$ that is obtained using data up to $T$. This approximation of national MLRG is reasonable in the middle of the sample over the period $[13, T-12]$. Since estimation of the last data point in the approximate bandpassed target is bad, in next subsections some national indicators to calculate MLRG are developed not only for the past but also in real time. Problem arises due to the fact that the national growth rate is observed only quarterly, while we are interested in a monthly indicator of economic activity, so that the interpolation is needed. Our results could be influenced by the interrelation among European and National bandpassed growths (see Table 3 below) and by the volatility in National bandpassed GDP (Table 4), as we will be using both national and European variables to calculate common factors. In the table below we show RMSE and correlation obtained by comparing National growth rate with Euro Area growth rate, smoothed by using a bandpass filter, see equation (7) above.

\begin{tabular}{l|c|c} 
& RMSE & Correlation \\
\hline \hline Italy & 0.31 & 0.77 \\
Belgium & 0.27 & 0.70 \\
France & 0.16 & 0.84 \\
Germany & 0.26 & 0.87 \\
Netherlands & 0.31 & 0.75 \\
Spain & 0.39 & 0.77 \\
\hline \hline
\end{tabular}

Table 3. European Bandpassed GDP versus National Bandpassed GDP in 1995-2008.

\begin{tabular}{l|c} 
COUNTRY & BANDPASSED GDP 1995,6-2008,1 \\
\hline \hline Spain & 0.05 \\
France & 0.08 \\
Italy & 0.14 \\
Germany & 0.16 \\
Netherlands & 0.16 \\
Belgium & 0.15 \\
Euro Area & 0.08 \\
\hline \hline
\end{tabular}

Table 4. Volatility in National bandpassed GDP.

Among the countries considered in this exercise, we observe the highest national volatility in bandpassed growth for Belgium, Germany, Italy, Netherlands smoothed growth rate; the 
lowest for Spain and France (this is similar to the one concerning Euro Area). France MLRG is, from Table 3 above, the more similar to Euro Area MLRG. In the following, we show for the considered countries results in a real time exercise concerning the three different approaches used to outline national indicators (we obtain three different indexes for each country). Real time estimates are built both from 2003 to 2008 and from 2003 to 2010, separately, because in the 2008-2010 period, we observe a strong recession and an high variation in volatility concerning GDP. We establish the criteria to apply in pseudo-real time evaluation to analyze the ability of $\hat{c}_{t}^{i}(t)$ for each $i$ country considered, to estimate (approximate) the national truncated band-pass filter $c_{t}^{* i}(T)$. Therefore, we analyze performance of the "National Eurocoin" at time $t$, with $t \leq T-12$, by the difference between our indicator at time $t$ and the approximate target at $\mathrm{t}$ that is obtained using data up to $T$.

Monthly length of the sample 2002-2010 is equal to $T=108$ for the period $T-96 \leq t \leq$ $T-13$. We are interested in:

(a). The RMSE of nowcast errors concerning the 2003,1,2009,12, period is outlined by the root of the ratio $\frac{\sum_{t=T-96}^{T-13}\left[\hat{c}_{t}^{i}(t)-c_{t}^{* i}(T)\right]^{2}}{84}$.

(b). The size of the revision errors after one month.

(c). The ability of $\hat{c}_{t}^{i}(t)-\hat{c}_{t-1}^{i}(t)=\Delta \hat{c}_{t}^{i}(t)$ to signal the correct change of the bandpassed variation $\Delta c_{t}^{* i}(T)$

(d). The analysis of turning points that we outline in next sub-section.

For every statistics outlined (points (a), (b), (c), (d) above) and for each country analyzed, we show the following results.

\begin{tabular}{l|ccc|ccc} 
& \multicolumn{3}{|c|}{$2003,1,2008,1$} & \multicolumn{3}{c}{$2003,1,2009,12$} \\
\hline COUNTRY & $\begin{array}{c}\text { Euro } \\
\text { GDP on } \\
\text { National Var. }\end{array}$ & $\begin{array}{c}\text { National } \\
\text { GDP on } \\
\text { National Var. }\end{array}$ & $\begin{array}{c}\text { National } \\
\text { GDP on } \\
\text { Euro Var }\end{array}$ & $\begin{array}{c}\text { Euro } \\
\text { GDP on } \\
\text { National Var. }\end{array}$ & $\begin{array}{c}\text { National } \\
\text { GDP on } \\
\text { National Var. }\end{array}$ & $\begin{array}{c}\text { National } \\
\text { GDP on }\end{array}$ \\
Euro Var. \\
\hline \hline Italy & 0.28 & 0.27 & 0.27 & 0.67 & 0.56 & 0.57 \\
Belgium & 0.29 & 0.31 & 0.30 & 0.59 & 0.68 & 0.51 \\
France & 0.19 & 0.17 & 0.12 & 0.44 & 0.37 & 0.32 \\
Germany & 0.37 & 0.38 & 0.39 & 0.69 & 0.63 & 0.62 \\
Netherlands & -- & -- & 0.29 & -- & -- & 0.59 \\
Spain & 0.44 & 0.22 & 0.10 & 0.47 & 0.43 & 0.44 \\
\hline \hline
\end{tabular}

Table 5. RMSE among Real Time Performance and National Bandpassed GDP.

In Table 5 above we consider the capacity of each national indicator in real time on nowcast of its bandpassed target. We observe that the projection of National GDP on European variables is generally the best strategy to adopt, followed by that of the projection of National GDP on National variables. In this section, it seems more useful and prudent to analyze performance in terms of RMSE from 2003 to 2008, before the unprecedented recession in 2008-2009 that strongly increases RMSE values. Since for Netherlands the number of variables in the Dataset useful for building common factors is very low (only 5 variables), it is 
A. Frenda, Journal Afrika Statistika, Vol. 7, 2012, pages 459-473. Short term forecasting of national growth.

impossible to build some "Netherlands common factors". Therefore the only strategy concerning the projection of National GDP on European variables is developed. Table 6 below shows that:

- Italy and France Indicator performance is particularly good in terms of revision error by the three different projection strategies;

- For Germany, Netherlands and Spain it is useful to project National GDP on Euro variables to minimize the revision error;

- Belgium shows a bad performance concerning the size of the revision errors.

\begin{tabular}{l|ccc}
\hline COUNTRY & $\begin{array}{c}\mid c \\
\text { Euro } \\
\text { GDP on } \\
\text { National Var. }\end{array}$ & $\begin{array}{c}\text { National } \\
\text { GDP on } \\
\text { National Var. }\end{array}$ & $\begin{array}{c}\text { National } \\
\text { GDP on } \\
\text { Euro Var }\end{array}$ \\
\hline \hline Italy & 0.030 & 0.033 & 0.026 \\
Belgium & 0.079 & 0.144 & 0.064 \\
France & 0.036 & 0.039 & 0.026 \\
Germany & 0.055 & 0.058 & 0.036 \\
Netherlands & - & - & 0.034 \\
Spain & 0.095 & 0.088 & 0.026 \\
\hline \hline
\end{tabular}

Table 6. Size of the revision errors after one month.

The null hypothesis of PT test Tables 7-9 is that the real time indicator (the predictor) and the (predicted) bandpassed target are independently distributed, i.e. the national Eurocoin, for each country, has no power in nowcasting the target. PT test, that is also used in testing Eurocoin approach for the whole Euro Area in Altissimo et al. (2010), is developed for the January 2003-December 2009 period for each country.

\begin{tabular}{l|ccc}
\hline COUNTRY & PT & $\begin{array}{c}\text { p-value of the } \\
\text { PT test statistic }\end{array}$ & $\begin{array}{c}\text { \% Correct prediction of } \\
\text { sign of bandpassed } \Delta\end{array}$ \\
\hline \hline Italy & 3.47 & 0.0000 & 0.69 \\
Belgium & 0.57 & 0.5704 & 0.53 \\
France & 2.57 & 0.0103 & 0.64 \\
Germany & 3.16 & 0.0016 & 0.67 \\
Netherlands & 3.20 & 0.0014 & 0.67 \\
Spain & 1.86 & 0.0628 & 0.59 \\
\hline \hline
\end{tabular}

Table 7. Non-parametric Statistic of PT: Strategy of the projection of National GDP on European variables.

By the projection of National GDP on Euro variables (Table 7), we observe that PT two sided test is: 
A. Frenda, Journal Afrika Statistika, Vol. 7, 2012, pages 459-473. Short term forecasting of national growth.

\begin{tabular}{l|ccc}
\hline COUNTRY & PT & $\begin{array}{c}\text { p-value of the } \\
\text { PT test statistic }\end{array}$ & $\begin{array}{c}\text { \% Correct prediction of } \\
\text { sign of bandpassed } \Delta\end{array}$ \\
\hline \hline Italy & 1.70 & 0.0895 & 0.58 \\
Belgium & -0.42 & 0.6752 & 0.48 \\
France & 0.98 & 0.3289 & 0.55 \\
Germany & 1.43 & 0.1517 & 0.58 \\
Netherlands & - & - & - \\
Spain & 0.31 & 0.7545 & 0.53 \\
\hline \hline
\end{tabular}

Table 8. Non-parametric Statistic of PT: Strategy of the projection of National GDP on National variables.

\begin{tabular}{l|ccc}
\hline COUNTRY & PT & $\begin{array}{c}\text { p-value of the } \\
\text { PT test statistic }\end{array}$ & $\begin{array}{c}\text { \% Correct prediction of } \\
\text { sign of bandpassed } \Delta\end{array}$ \\
\hline \hline Italy & 1.94 & 0.0524 & 0.60 \\
Belgium & 1.08 & 0.2807 & 0.57 \\
France & $1.87^{*}$ & 0.0608 & 0.60 \\
Germany & 1.38 & 0.1685 & 0.58 \\
Netherlands & - & - & - \\
Spain & 1.22 & 0.2212 & 0.58 \\
\hline \hline
\end{tabular}

* We obtain a PT equal to 2.15 for the period 2003,1-2010,12

Table 9. Non-parametric Statistic of PT: Strategy of the projection of National GDP on National variables.

- Above $99 \%$ critical value for Italy (particularly), Germany and Netherlands.

- Above 95\% critical value for France.

For Spain and Belgium we observe a lower performance in terms of correct prediction of sign; particularly for Belgium we observe that the significant level of the test is very low (very high p-value).

The projection of National GDP on National variables (Table 8) shows a better performance for Italy and for Germany in terms of prediction of sign (0.58\%).

By the projection of the European GDP on National variables (Table 9), we observe that PT test is above $90 \%$ and near $95 \%$ critical value for Italy and France. For these countries we observe a sufficient prediction of signs in the bandpassed target.

\section{Some conclusions:}

Analyzing real time performance in 2003-2008, we observe that:

- In terms of RMSFE national indicators concerning Italy, France and Spain provide a fairly good approximation of band-pass GDP in real time, particularly by the projection of National GDP on the 157 European variables included in the dataset. 
- During the 2008-2010 period, we observe a very high volatility that doesn't allow the obtaining of a clear comparison of our indicators. For France, RMSFE is particularly good, considering business cycle volatility (in this phase). The interrelations among a large dataset of European variables and National gross domestic product seem to better our estimates.

- Also, in terms of size of revision, Italy and France perform better than other countries.

- Concerning the ability of the real time indicator to track target direction, we show that Italy, France, Germany, Netherlands perform fairly well by the projection of National GDP on Euro variables.

The figures below show, for each country, real time performance, obtained by using Eurocoin methodology and the three strategies outlined above.
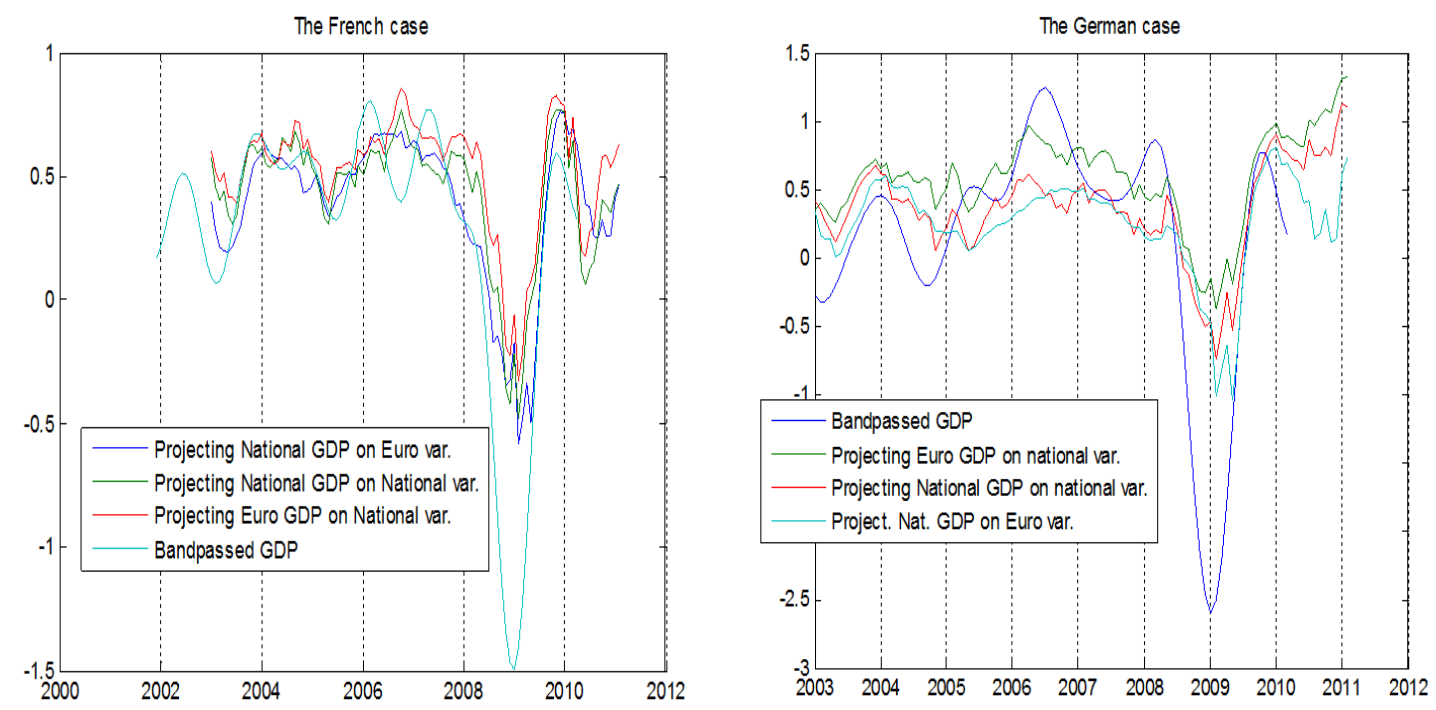

Fig. 1. Comparing National Real Time Indicators with Bandpassed GDP

It seems interesting to observe that real time estimation is generally not able to detect the depth of the recession in 2008-2010: e.g. for Italy (in the figure below) the minimum of the monthly quarter on quarter GDP growth rate in real time is equal to -0.9 , while for the bandpassed target we have a minimum value of - 2.4.

\subsection{Behaviour around Turning Points}

A characteristic of these national indicators is the ability to give a correct signal of MLRG turning points (TP) in real time. We may say that an upturn (downturn) signal in can be predicting or lagging true TP (i.e. a four-month error is tolerated). We outline the TP for each strategies considered in the national experiment by the Bry-Boschan procedure: 


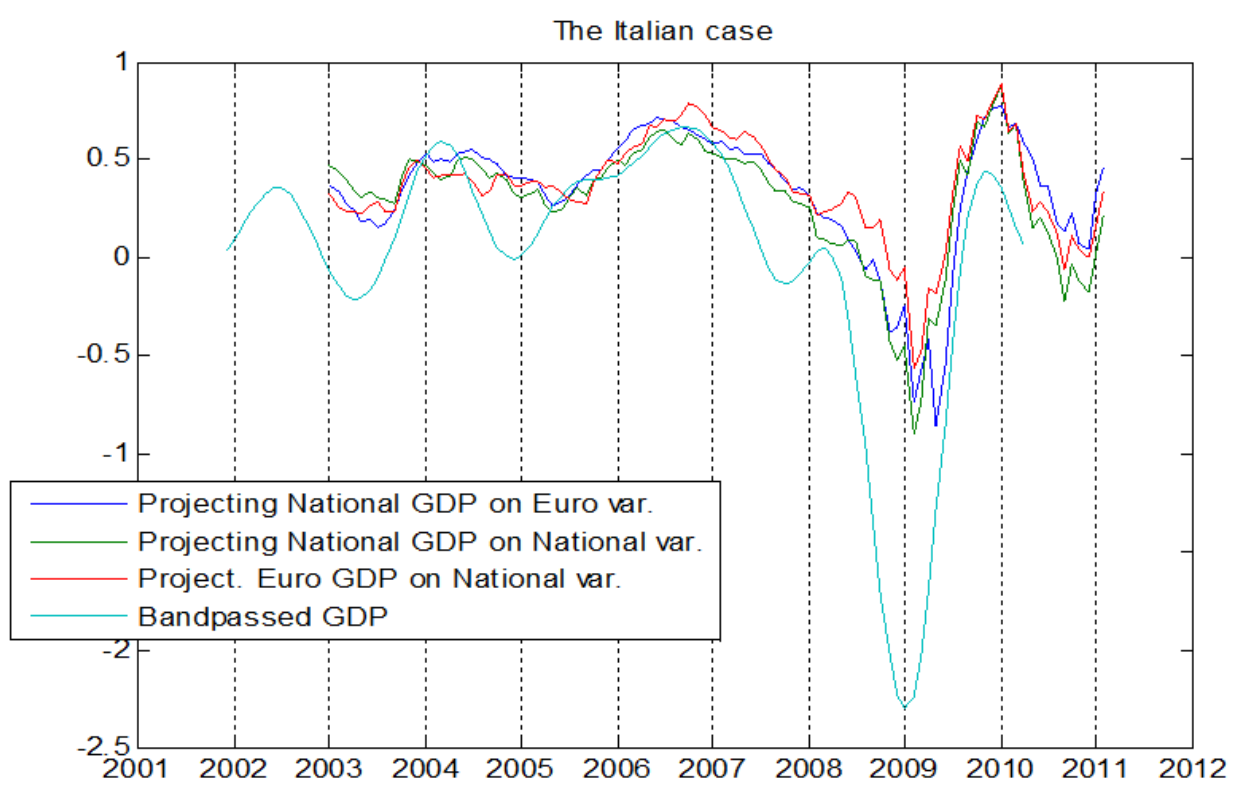

Fig. 2. National Real Time Indicators versus Bandpassed GDP

\begin{tabular}{l|ccc} 
COUNTRY & TOTAL TURNING POINTS & DOWNTURNS & UPTURNS \\
\hline \hline Italy & 4 & 2 & 2 \\
Belgium & 4 & 2 & 2 \\
France & 4 & 2 & 2 \\
Germany & 4 & 2 & 2 \\
Netherlands & 4 & 2 & 2 \\
Spain & 5 & 3 & 2 \\
\hline \hline
\end{tabular}

Table 10. Number of Turning Points in the Bandpassed Target.

We observe that real time indicators for Italy and France are able to detect the most part of $\mathrm{TP}$ in the target.

\section{Main Conclusions}

This work contributes to the debate on the estimation of smoothed growth by generalized dynamic factor models. Eurocoin indicator, published monthly by the Bank of Italy and CEPR, provides a summary index of the MLRG only for the whole Euro area aggregate, therefore it is an aggregate indicator. The innovation of this work are some disaggregated procedures based on Eurocoin approach, to give a monthly smoothed estimate of quarteron-quarter growth rate with regard to the European countries. The Eurocoin approach is 
A. Frenda, Journal Afrika Statistika, Vol. 7, 2012, pages 459-473. Short term forecasting of national growth.

\begin{tabular}{l|cccc} 
COUNTRY & TP Signals & Correct TP & Correct over signalled TP & Missed over all TP \\
\hline \hline Italy & 4 & 3 & $3 / 4$ & $1 / 4$ \\
Belgium & 4 & 2 & $2 / 4$ & $2 / 4$ \\
France & 4 & 3 & $3 / 4$ & $1 / 4$ \\
Germany & 4 & 2 & $2 / 4$ & $2 / 4$ \\
Netherlands & 4 & 4 & $4 / 4$ & - \\
Spain & 4 & 2 & $2 / 4$ & $3 / 5$ \\
\hline \hline
\end{tabular}

Table 11. Real time detection of turning points (TP).

implemented in this thesis to estimate monthly smoothed GDP components for the following reasons:

1. Euro Area flash estimates are released on a quarterly frequency, with a certain delay (8-10 weeks).

2. GDP growth in any quarter depends on seasonal effects and measurement errors. On the contrary, Eurocoin methodology highlights the underlying trend by adjusting growth rate for short-term fluctuations and measurement errors.

3. Our disaggregated indicators can be influenced by factors affecting only a particular country, differently from the classic Eurocoin indicator.

4. An alternative to Eurocoin in summarizing the current economic picture and in tracking underlying growth for Euro area could be the band-pass filters, but they can deteriorate at the end of the sample, and they are less reliable for the most recent data. Differently, Eurocoin and the disaggregated indicators that we outline in this research extract information relevant to the short-term forecasting of GDP, preceding official releases and bandpass filters by several months.

By the statistics developed in this paper, it is clear that by applying Eurocoin methodology based on dynamic factor model and by using European and National variables contained in Thomson Financial Datastream (TFD), in particular for Italy and France we can obtain some national indicators with a good performance that can compete with the truncated band-pass filters. In addition, they do not deteriorate at the end of the sample. The strategy used in obtaining these national performances is projecting National GDP on the European common factors that are a linear combination of the 157 European variables included in the dataset. However, we obtain interesting results for Italy and Germany by projecting National GDP on National common factors (e.g. by the projection of German GDP on a linear combination of the observable German monthly data in the TFD): these could mean that, for some countries, the forecasts of the medium to long run component of GDP is mostly influenced by domestic factors.

In synthesis, it may be possible to estimate the month-on-month changes of the smoothed GDP (unobservable) both by using a large dataset with 157 variables in the case of the TFD and also by a more parsimonious number of variables. Clearly, such conclusions are not general but restricted to the data and the models used in these exercises.

Journal home page: www.jafristat.net 
A. Frenda, Journal Afrika Statistika, Vol. 7, 2012, pages 459-473. Short term forecasting of national growth.

\section{References}

Altissimo, F., Bassanetti, A., Cristadoro, R., Forni, M., Hallin, M., Lippi, M., Reichlin, L. and Veronese, G., 2001. A real Time Coincident Indicator for the euro area Business Cycle. CEPR Discussion Paper No. 3108.

Altissimo, F., Cristadoro, R., Forni, M., Lippi, M. and Veronese, G., 2010. New Eurocoin: tracking economic growth in real time. The Review of Economics and Statistics, MIT Press, vol. 92(4), 1024-1034.

Backus D.K. and Kehoe, P.J., 1992. International Evidence on the Historical Properties of Business Cycles. The American Economic Review, 82, 864-888.

Bai, J. , 2003. Inferential theory for factor models of large dimension. Econometrica 71, 135-71.

Bai, J. and Ng, S., 2002. Determining the number of factors in approximate factor models. Econometrica 70, 191-221.

Baxter, A. and King, R.G., 1999. Measuring Business Cycle Approximate Band-Pass filters for Economic Time Series. The Review of Economics and Statistics 81, 575-93.

Burns, A.F., e Mitchell W.C., 1946. Measuring Business Cycle, New York, NBER.

Chadha, J.S., Janssen, N. and Nolan, C., 2000. An examination of UK business cycle fluctuations: 1871-1997. Discussion paper in economics and management, University of Reading.

Christiano, L.J. and Fitzgerald, T.J., 1999. The Band Pass Filter, NBER Working Paper No. W7257.

Christiano, L.J. and Fitzgerald, T.J., 2003. The Band-Pass Filter. International Economic Review 84, 435-65.

Cristadoro, R., Forni, M., Reichlin, L. and Veronese, G., 2005. A core inflation indicator for the euro area, Journal of Money Credit and Banking 37(3), 539-560.

D'Ambra, A., Sarnacchiaro, P., 2010. Some data reduction methods to analyze the dependence with highly collinear variables: a simulation study. Asian Journal of Mathematics and Statistics 3(2): 69-81.

D'Ambra, L., Sabatier, R. and Amenta, P., 2001. Three way factorial analysis: synthesis and new approaches. Italian Journal of Applied Statistics, 13, 101-117.

European Commission, 1997. The joint Harmonized EU program of Business and Consumer Surveys, European Economy, $\mathrm{n}^{\circ} 6$.

orni, M., Hallin, M. Lippi, M. and Reichlin, L., 2000. The generalized dynamic factor model: identification and estimation. The Review of Economics and Statistics 82, 540-554.

Forni, M., Hallin, M. Lippi, M. and Reichlin, L., 2001. Coincident and leading indicators for the euro area. The Economic Journal 111, 62-85.

Forni, M., Hallin, M. Lippi, M. and Reichlin, L., 2004. The generalized dynamic factor model: consistency and rates. Journal of Econometrics 119, 231-255.

Forni, M., Hallin, M. Lippi, M. and Reichlin, L., 2005. The generalized dynamic factor model: one-sided estimation and forecasting. Journal of the American Statistical Association 100 830-40.

Forni, M. and M. Lippi, 2001. The generalized dynamic factor model: representation theory. Econometric Theory 17, 1113-1141.

Frenda, A., 2011. Analyzing National smoothed growth by generalized dynamic factor model. International Journal on GSTF Business review, Vol.1, No.1. 
A. Frenda, Journal Afrika Statistika, Vol. 7, 2012, pages 459-473. Short term forecasting of national growth.

Frenda, A., 2011. Empirical Applications: Disaggregating a Dynamic Factor Model - Interdisciplinary Workshop: Econometric and Statistical Modelling of Multivariate Time Series-Louvain-la-Neuve 25-27 Maggio.

Frenda, A., 2012. Estimating smoothed GDP components. China-USA Business Review, David Publishing Company, Vol. 11, No.1.

Hallin, M. and Liska, R., 2007. Determining the Number of Factors in the Generalized Factor Model, Journal of the American Statistical Association, 102(478), 603-617.

Stock, J.H. and Watson, M.W., 2002. Forecasting using principal components from a large number of predictors. Journal of the American Statistical Association 97, 1167-79. 